\title{
Macroscopic tensile plasticity of Zr-based bulk metallic glass with surface screw thread shaped structure
}

\author{
M. Gao ${ }^{\mathrm{a}}$, C. Wang ${ }^{\mathrm{a}}$, J. Dong ${ }^{\mathrm{b}}$, Y. Huan ${ }^{\mathrm{b}}$, H. Y. Baia and W. H. Wang, ${ }^{\mathrm{a}, *}$ \\ 'Institute of Physics, Chinese Academy of Sciences, Beijing 100190, People's \\ Republic of China \\ ${ }^{b}$ State Key Laboratory of Nonlinear Mechanics (LNM), Institute of Mechanics, \\ Chinese Academy of Sciences, Beijing 100190, China
}

Zr-based bulk metallic glass (BMG) dog-bone-shaped tensile specimens with designed screw thread shaped (STS) structures fabricated by mechanical turning were tested under uniaxial tensile loading. Obvious macroscopic tensile plasticity and serrated flow behavior appear when the typical size of STS structures-the depth reaches the intrinsic plastic zone size for MGs. Finite element analysis show the introduced STS structure twists the stress field distribution and then hampers the main shear band propagation and promotes the formation of multiple shear bands, which improve the tensile plasticity. The proposed STS designing scheme may shed light on the deformation mechanism and be helpful for structural application of BMGs.

Keywords: Bulk metallic glass, surface screw thread shaped structure, macroscopic tensile plasticity, multiple shear bands, stress field torsion

* Corresponding author. Tel.: +86 10 82649573; fax: +86 10 82640223; e-mail: whw@iphy.ac.cn 


\section{Introduction}

Bulk metallic glasses (BMGs) have been extensively studied as potential engineering and functional materials since the materials have excellent mechanical and physical properties compared to their crystalline counterparts. The long-range disordered atomic structure and metallic bonding render the BMGs ultrahigh-yield strength, large elastic strain limit, ultrahigh fracture toughness, high corrosion resistance, excellent soft magnetic properties and the ability to be processed like a plastic [1-4]. On the other hand, these intrinsic structural characteristic of BMGs usually arise the local softening under loading and then directly lead to the catastrophic brittle fracture with nearly zero global tensile plasticity, which is the major obstacle for their structural applications [5-7]. Although some BMG composites with large tensile plasticity have been successfully developed by introducing the second crystalline phases [8-9], monolithic BMGs show poor macroscopic plasticity under uniaxial tensile loading, which remains a big challenge.

At room temperature, plastic deformation of BMGs under the external loading is highly localized into a narrow shear band (SB) with the width of $10 \mathrm{~nm}$. As shearing deformation proceeds, the friction heat and the drastically reduction of viscosity within the SB strongly weaken the load capacity of MGs, arising the "work softening" behaviors and subsequently catastrophic fracture [10]. Yet the development of such SBs does not necessarily result in catastrophic fracture. For example, when these SBs are spatially confined under certain loading states, such as the compressive and bending loading, global plasticity has been improved by forming a large number of SBs and stabilizing the propagation of SBs [11-12]. Particularly, various BMGs actually own the local plastic deformation ability even for the brittle Mg-, La-based BMGs [13-14]. A question is then raised: How could we extend the local plastic deformation into the macroscopic tensile plasticity rather than the brittle fracture governed by a thin main SB? Recent work showed that the macroscopic tensile

plasticity could be obtained by introducing the artificial micro-structural defects, such as the notches [15-16], the surface indentations [17], the designing hole array [18] and 
laser shock peening treatment [19]. These results indicate that the formation of multiple SBs and the stable propagation of main SBs are the key factor for improving the tensile plasticity of BMGs.

In present work, we proposed a novel surface treatment on the BMG to activate the formation of multiple SBs. Two BMG dog-bone-shaped tensile specimens with different screw thread shaped (STS) structures obtained by mechanical turning were fabricated. Under uniaxial tensile loading, obvious macroscopic tensile plasticity and serrated flow behavior appear when the typical size of STS structures-the depth reaches the intrinsic crack tip plastic zone size. We applied the finite element simulations to analyze the difference of the stress field distribution and the resulting macroscopic tensile plasticity for the BMG tensile specimens with different STS structures.

\section{Experimental}

Typical Zr-based BMG with a nominal chemical composition of $\mathrm{Zr}_{64.13} \mathrm{Cu}_{15.75} \mathrm{Ni}_{10.12} \mathrm{Al}_{10}$ was chosen as the model material for its good glass-forming ability and excellent mechanical properties [11]. The $3 \mathrm{~mm}$ BMG rods were prepared from a master alloy by water-cooled copper mold casting. Its glassy nature was

checked by X-ray diffraction and differential scanning calorimetry. Dog bone-like specimens for tensile tests with gauge diameter of $1.5 \mathrm{~mm}$ and gauge length of $10 \mathrm{~mm}$ were cut from the BMG rods using electric spark line cutting machine. All tensile specimens were polished with $1.5 \mu \mathrm{m}$ diamond sandpaper to get rid of corrosion pits induced by electric spark line cutting.

Before tensile tests, the STS was introduced in the middle of the dog bone-like specimens by mechanical turning, as is shown in Fig. 1a. The mechanical clamp is made of the stainless steel and the turning tool is made of cubic boron nitride material. The fabrication process of the STS is very simple. Firstly, one end of the MG rod is 
fixed in the lathe by the mechanical clamp and the other end of the MG rod is free for moving along the forward direction (as is shown by the blue arrow in Fig. 1a). Secondly, the structural parameters of the STS are designed and are entered into the turning controlling system. Finally, the turning tool is controlled by the controlling system to fabricate the STS on the surface of the MG rod. To study the influence of the STS structure size on the tensile mechanical behaviors, we specially designed two different STS specimens: I and II. Previous research showed that the depth of the introduced notch largely affects the mechanical behaviors of MGs [16]. Thus, in this work, we mainly studied the effect of the key factor-the depth of the STS structures in the tensile behaviors. And the influence of the width, spacing and inclination angle of the STS structures on the tensile mechanical behaviors will be the research focus in future. The detailed structural parameters are listed in Table 1. In Fig. 1b, as an example, the surface morphology of the final fabricated STS specimen II is displayed. From the inserted graph of Fig.1b, one can clearly see that the fabricated specimen still keeps the amorphous nature confirmed by X-ray diffraction after mechanical turning. The introduced STS structure has the average dimensions, $35 \times 71 \times 74 \mu \mathrm{m}^{3}$ (depth $\times$ width $\times$ spacing), and the inclination angle of $7.5^{\circ}$ with respect to the normal direction (Fig. 1c). Fig. 1d and Fig. 1e show the detailed surface morphology of the groove portion (marked by A) and raised portion (marked by B) of STS structure in Fig. 1c. One can see clearly that the hierarchical microstructure on the surface of part $\mathrm{A}$ and $\mathrm{B}$ originating from the mechanical turning and no pre-SBs appear in the specimen. Uniaxial tensile tests were conducted on the as-cast and STS BMG specimens with a constant quasi-static strain rate of about $1 \times 10^{-4} \mathrm{~s}^{-1}$ under an INSTRON ElectroPuls E10000 All-Electric Test Instrument at room temperature. Tensile strain was precisely and directly measured based on the sample gauge length using non-contacting video extensometer (INSTRON). At least three specimens were measured to ensure the reproducibility. The fracture features, such as newly generated tensile fracture surfaces, fracture side surface morphology and fracture angle, were observed by the scanning electron microscopy (SEM) conducted in a Philips XL30 instrument. 


\section{Results and discussion}

3.1. Engineering tensile stress and tensile strain curves for as-cast and STS BMG specimens

Fig. 2 shows the typical engineering tensile stress and strain curves for as-cast and STS BMG specimens. For the as-cast specimen, besides the linear elastic deformation, no visible macroscopic tensile plasticity and the catastrophic fracture takes place when the tensile strain reaches about $2 \%$. For the STS specimen I with the smaller STS structure depth, there appears a small nonlinear trend and the fracture strength does not change compared to that of the as-cast one. In contrast, for the STS specimen II, the tensile stress and strain curve becomes nonlinear when the tensile strain increases into $1.37 \%$ and then obvious yielding behavior appears. The enlarged portions of the tensile stress-strain curves for as-cast and STS specimens at high stress level are displayed in the inserted graph of Fig. 2. For STS specimen II, the macroscopic tensile plastic strain is about $0.90 \%$ and the tensile fracture strength is about $1506 \mathrm{MPa}$, which is slightly smaller than that of the as-cast one (1614 MPa). On the other hand, it is obviously found that the STS specimen II displays the serrated flow behavior in its stress-strain curve compared to that of the brittle fracture of the as-cast specimen and STS specimen I. The dense and large serrated flow events of the STS specimen II indicate that the deformation process is mainly governed by the discontinuous and intermittent dominant $\mathrm{SB}$ propagation process, and then considerable plastic deformation through shear banding should be produced during tensile stretching $[2,20]$.

This can be further confirmed by observing surface morphology of the specimens after fracture as is shown in Fig. 3. Fig. 3a shows that there is almost no SBs on the surface of as-cast specimen and catastrophic fracture takes place by main SB fast slipping along the main shear plane. For STS specimen I in Fig. 3b, there is only main shear band and the brittle fracture is the main fracture mode. In contrast, for STS specimen II in Fig. 3c, a lot of SBs appear in the groove portion of STS 
structure, as is shown in the central part of Fig. 3c. Therefore, the accordance between the nonlinear tensile stress-strain curve and the surface morphology demonstrates that the tensile plasticity obtained in monolithic BMGs by surface STS treatment is contributed from STS structure II by activation of multiple SBs.

3.2. SEM observations of sample surface and fracture surface for as-cast and STS BMG specimens

The STS treatment also induces considerable change in fracture angle and fracture morphology of BMGs. As is shown in the left part of Fig. 3a, the as-cast sample fails by a single main shear fracture, with a shear fracture angle of about $53^{\circ}$, which is consistent with the previous results [21]. For the STS specimen I with the smaller STS structure depth in Fig. 3b, the fracture angle is about $55^{\circ}$ and it is close to that of as-cast one. In contrast, the STS specimen II exhibits the larger fracture angles $\left(66.1^{\circ}\right.$, as shown in the left part of Fig. 3c) than that of as-cast one, which implies that the introduced STS structure indeed changes the propagation path of main SBs during tensile deformation. What is more, the fracture surface of STS specimen II seems rougher than that of as-cast specimen and STS specimen I (the left parts of Fig. 3), which is ascribed to the torsion effect of STS structure on the main shear plane. Typical fracture morphology also displays a difference as is shown in the right parts of Fig. 3. Compared to the actinomorphic fracture morphology of as-cast specimen and STS specimen I, the STS specimen II exhibits a complex morphology consisting of the river-like pattern and the similar radial-like pattern. Previous research indicates that the radial pattern under uniaxial tensile loading shows the dominant role of the normal stress in fracture process of BMGs [21]. For STS specimen II, the river-like pattern appearance reveals the shear stress involvement in the deformation and fracture process [22]. The above experimental results further show that besides the activation of the promotion of the multiple SBs, the STS structures of STS specimen II impede the original main SBs propagation and twist the main shear plane into a plane being more difficult to fast slip, which promotes the improvement of the 
macroscopic tensile plasticity. Compared to the STS specimen II, the STS structure of STS specimen I has the smaller depth and have a little effect in the tensile mechanical behaviors. It implies that it is the STS structures with the special spatial length that improve the tensile plasticity. All of the above tensile mechanical properties for as-cast and STS specimens are listed in Table 1.

\subsection{Finite element analysis of the stress field distribution for STS BMG specimens}

To further investigate the deformation mechanism of the STS BMG specimens during the tensile deformation and the influence of the introduced STS structure on the stress field distribution, the finite element analysis based on the elastic-perfectly plastic model due to no work-hardening for most BMGs [2] was performed. It should be noted that the finite element model in this study is the standard code of the elastic-perfectly plastic solid without previously setting the structural type and the pre-existing structural defects. Fig. 4a displays the whole stress field distribution of the STS specimen II (left part) and I (right parts) under uniaxial tensile loading. One can see that the stress concentration phenomenon appears in the groove portion of STS structure along the screw circumferential direction for both STS specimens. For STS specimen II, the stress concentration zones have a larger size than that of STS specimen I. Thus, these larger stress concentration zones in STS specimen II usually tend to connect and form the SBs due to the geometric restrictions, which is similar to the stress distribution under the bending and compression loading [23-24] and then leads to the formation of multiple SBs as shown in Fig. 3c. However, for the STS specimen I, the smaller stress concentration zones do not arouse the formation of other SBs except for the main SB and then do not impede the propagation of main SB. Fig. $4 \mathrm{~b}$ shows the longitudinal sectional view of the stress field direction distribution for STS specimens. For STS specimen I in the right part of Fig. 4b, the stress field displays the homogeneous and similar distribution in both the STS-structure part and non-STS-structure part. In contrast, for STS specimen II in the left part of Fig. 4b, there indeed exists the stress field deviation from the external tensile loading direction 
near the STS structure, which directly leads to the above larger stress concentration. The transverse sectional view in the left part of Fig. $4 \mathrm{c}$ more clearly shows the stress field deviation. The first picture marked 1 gives the stress field distribution of the transverse section of STS structure corresponding to part 1 in the left part of Fig. 4a and the stress field distribution is parallel to the external loading direction. Surprisingly, from the second picture to forth picture, the stress field gradually produces a deflection dependent on the geometry shape of the introduced STS structure. In contrast, for STS specimen I, there is no stress field deviation in the right parts of Fig. $4 \mathrm{~b}$ and $4 \mathrm{c}$. Thus, the introduced STS structures in the STS specimen II actually produce an equivalent torsion effect in the whole stress field. This STS structure induced twisted stress field is analogous to the stress field under torsion loading and the torsion stress is actually the restricted stress field. Under the twisted stress field, multiple SBs formation could be easily promoted, the main SB propagation could be stabilized, and then contributes to the occurrence of macroscopic plastic deformation [25-26].

Above finite element analysis further confirms that the designed STS structure induced the equivalent twisted stress field brings about the macroscopic tensile plasticity in the BMG. What is more, it is noted that this mechanism of improving the tensile plasticity for BMGs is different from the tensile plastic deformation mechanism governed by dislocation slipping in crystalline materials. For crystalline materials, when applying the external loading, the intrinsic structural defects-dislocations undertake the most of stress and then the plastic deformation take places by slipping or twinning of dislocations. Especially for metallic crystals, under the applied stress, the initial dislocations are easily started by the metallic atomic sliding on the sliding planes. After the "seed" of the initial dislocations appears, these dislocations are prone to propagate in the way of the slipping and twinning and the permanent plastic flow takes place. In contrast, for BMGs, there exists nano-scale structural heterogeneity-flow units [27-28]. Under external loading, plenty of flow units are activated along the main shear plane and then forms the main SB [29]. When 
reaching the yielding, the single main SB is easy to fast propagate and then directly leads to the brittle fracture without tensile plasticity. Our methods just introduced the surface screw thread shaped structures to induce the formation of multiple SBs and prevent the fast propagation of the main SB.

We note that one could obtain bendable and malleable macro-sized glasses through residual stress engineering [30]. However, it is difficult to realize in practice considering that there is no clear regulating standards and no definite physical parameter to reach the optimal effect for the residual stress controlling. Recent research on the enhancement of the tensile plasticity by introduction of the artificial heterogeneous microstructures [16-18, 23] have confirmed the feasibility of the artificial surface geometric treatment. It has been verified that the stabilization of shear band propagation and crack opening require that the typical length of the artificial heterogeneous microstructures $L<R_{P} . R_{P}$ is the intrinsic crack tip plastic zone radius, and $R_{P} \sim(1 / 2 \pi)\left(K_{I C} / \sigma_{y}\right)^{2}$, here $K_{I C}$ is fracture toughness and $\sigma_{y}$ is the yield strength) $[8,13]$, which is a key parameter governing the fracture mode. In this perspective, the intrinsic crack tip plastic zone radius could be considered as the definite physical parameter of the surface geometric treatment. In our work, for the STS specimen II, the depth of the STS microstructure are $71 \mu \mathrm{m}$. This is comparable to the value of $R_{p}$ for $\mathrm{Zr}$-based MGs (about $100 \mu \mathrm{m}$ ), which is prone to form multiple SBs. However, for the STS specimen I, the depth of of the STS microstructure is just only $17 \mu \mathrm{m}$, which is much lower that $R_{P}$, and the tensile mechanical property almost have no change. Therefore, based on the above analysis, the validity of STS treatment in the improvement of the tensile plasticity depends on the intrinsic structural characteristics of MGs, the intrinsic crack tip plastic zone size $R_{P}$. And the optimal depth of the STS structures should be chosen for different values in different MG systems. In addition, the surface geometric treatment method such as our proposed STS method is relatively easier to control and tune in engineering fields. Our present strategy could enlighten more artificial surface geometric treatment methods with controllable design for toughing BMGs. 


\section{Conclusions}

Macroscopic tensile plasticity for monolithic BMGs is obtained by the special surface geometric treatment of STS. The introduced STS structures promote the formation of multiple SBs and prevent the fast propagation of main SBs. Finite element analysis shows that the stress field of the STS MG specimen under uniaxial tensile loading is twisted and is analogous to the twisted stress field with geometric restraint characteristic. We note that the effectiveness of the present method closely depends on the unique structural characteristics of BMGs and the deformation mechanism, that is, the typical size of the surface geometric treatment should be selected based on the intrinsic crack tip plastic zone radius.

\section{Acknowledgments}

The work is supported by the NSF of China (Grant No. 51271195, 51461165101 and 11372323) and the MOST 973 Program (No. 2015CB856800). The discussions and experimental help of D. Q. Zhao, and valuable discussions with Prof. M. X. Pan are appreciated.

\section{References}

[1] W. L. Johnson, Bulk glass-forming metallic alloys: Science and technology, MRS Bull. 24 (1999) 42-56.

[2] C. A. Schuh, T. C. Hufnagel, U. Ramamurty, Mechanical behavior of amorphous alloys, Acta Mater. 55 (2007) 4067-4109.

[3] A. Inoue, Bulk amorphous and nanocrystalline alloys with high functional properties, Mater. Sci. Eng., A 304-306 (2001) 1-10.

[4] M.D. Demetriou, M. E. Launey, G. Garrett, J. P. Schramm, D. C. Hofmann, W. L. Johnson, R. O. Ritchie, A damage-tolerant glass, Nat. Mater. 10 (2011) 123-128. 
[5] T. Mukai, T. G. Nieh, Y. Kawamura, A. Inoue, K. Higashi, Dynamic response of a $\mathrm{Pd}_{40} \mathrm{Ni}_{40} \mathrm{P}_{20}$ bulk metallic glass in tension, Scr. Mater. 46 (2002) 43-47.

[6] Z. F. Zhang, J. Eckert, L. Schults, Difference in compressive and tensile fracture mechanisms of $\mathrm{Zr}_{59} \mathrm{Cu}_{20} \mathrm{Al}_{10} \mathrm{Ni}_{8} \mathrm{Ti}_{3}$ bulk metallic glass, Acta Mater. 51 (2003) 1167-1179.

[7] J. R. Greer, J. T. M. De Hosson, Plasticity in small-sized metallic systems: Intrinsic versus extrinsic size effect, Prog. Mater. Sci. 56 (2011) 654-724.

[8] D. C. Hofmann, Jin-Yoo. Suh, A. Wiest, G. Duan, M. L. Lind, M. D. Demetriou, W. L. Johnson, Designing metallic glass matrix composite with high toughness and tensile ductility, Nature 451 (2008) 1085-1089.

[9] Y. Wu, Y. Xiao, G. Chen, C. T. Liu, Z. Liu, Bulk metallic glass composite with transformation-mediated work-hardening and ductility, Adv. Mater. 22 (2010) $2770-2273$.

[10] J. J. Lewandowski, A. L. Greer, Temperature rise at shear bands in metallic glasses, Nat. Mater. 5 (2006) 15-18.

[11] Y. H. Liu, G. Wang, R. J. Wang, D. Q. Zhao, M. X. Pan, W. H. Wang, Super plastic bulk metallic glasses at room temperature, Science 315 (2007) 1385-1388.

[12] B. Sarac, J. Ketkaew, D. O. Popnoe, J. Schroers, Honeycomb structures of bulk metallic glasses, Adv. Funct. Mater. 22 (2012) 3161-3169.

[13] X. K. Xi, D.Q. Zhao, M.X. Pan, W.H. Wang Y. Wu, J. J. Lewandowski. Fracture of brittle metallic glasses: brittleness or plasticity, Phys. Rev. Lett. 94, (2005) 125510 .

[14] F. F. Wu, Z. F. Zhang, S. X. Mao, Size-dependent shear fracture and global tensile plasticity of metallic glasses, Acta Mater. 57 (2009) 257-266.

[15] R. T. Qu, M. Calin, J. Eckert, Z. F. Zhang, Metallic glasses: notch-intensive materials, Scr. Mater. 66 (2012) 733-736.

[16] Z. T. Wang, J. Pan, Y. Li, C. A. Schuh, Densification and strain hardening of a metallic glass under tension at room temperature, Phys. Rev. Lett. 111 (2013) 135504. 
[17] R. T. Qu, Q. S. Zhang, Z. F. Zhang, Achieving macroscopic tensile plasticity of monolithic bulk metallic glass by surface treatment, Scr. Mater. 68 (2013) $845-848$.

[18] B. Sarac, J. Schroers, Designing tensile ductility in metallic glasses, Nat. Comm. 4 (2013) 2158.

[19] Q. Wang, Y. Yang, H. Jiang, C. T. Liu, H. H. Ruan and J. Lu, Superior tensile ductility in bulk metallic glass with gradient amorphous structure, Sci. Rep. 4 (2015) 4757.

[20] B. A. Sun, H. B. Yu, W. Jiao, H. Y. Bai, D. Q. Zhao, W. H. Wang, Plasticity of ductile metallic glasses: a self-organized critical state, Phys. Rev. Lett. 105 (2010) 035501 .

[21] Z. F. Zhang, G. He, J. Eckert, L. Schultz, Fracture mechanisms in bulk metallic glassy materials, Phys. Rev, Lett. 91 (2003) 045505.

[22] B.A. Sun, W.H. Wang, The fracture of bulk metallic glasses, Prog. Mater Sci. 74 (2015) 211-307.

[23] R. T. Qu, J. X. Zhao, M. Stoica, J. Eckert, Z.F. Zhang, Macroscopic tensile plasticity of bulk metallic glass through designed artificial defects, Mater. Sci. Eng., A 534 (2012) 365-373.

[24] B. Sarac, B. Klusemann, T. Xiao, S. Bargmann, Materials by design: an experimental and computational investigation on the microanatomy arrangement of porous metallic glasses, Acta Mater. 77 (2014) 411-422.

[25] X. Q. Lei, Y. J. Wei, B. C. Wei, W. H. Wang, Spiral fracture in metallic glasses and its correlation with failure criterion, Acta Mater. 99 (2015) 206-212.

[26] Z. Kovacs, M. Ezzeldien, K. Mathis, P. Ispanovity, F. Chmelik, J. Lendvai, Statistical analysis of acoustic emission events in torsion deformation of a Vitreloy bulk metallic glass, Acta Mater. 70 (2014) 113-122.

[27] Y. H. Liu, D. Wang, K. Nakajima, W. Zhang, A. Hirata, T. Nishi, A. Inoue, M. W. Chen, Characterization of nanoscale mechanical heterogeneity in a metallic glass by dynamic force microscopy, Phys. Rev. Lett. 106 (2011) 125504.

[28] H. Wagner, D. Bedorf, S. Kuchemann, M. Schwabe, B. Zhang, W. Arnold, K. 
Samwer, Local elastic properties of a metallic glass, Nat. Mater. 10 (2011) 439-442.

[29] W. H. Wang, Y. Yang, T. G. Nieh, C. T. Liu, On the source of plastic flow in metallic glasses: concepts and models, Intermetallics 67 (2015) 81-86.

[30] Y. Zhang, W. H. Wang, A. L. Greer, Making metallic glasses plastic by control of residual stress, Nat. Mater. 5 (2006) 857-860.

\section{Figures captions}

Fig. 1. (a) Illustration of STS structure fabrication process by mechanical turning on the surface of MG rod-like specimen. (b) The dog-bone-shaped tensile STS specimen II. Blue dashed rectangular marks the screw thread shaped part and the inserted graph gives the experimental confirmation of the amorphous nature after mechanical turning by X-ray diffraction. (c) High-magnification detailed microgragh corresponding to the part marked by the blue-dashed rectangular in Fig. 1b. (d) Detailed microgragh of the part $A$ pointed by the black arrow in Fig. 1c. (e) Detailed microgragh of the part B pointed by the black arrow in Fig. 1c.

Fig. 2. Typical engineering tensile stress-strain curves of both as-cast specimen and STS specimen I and II. Inserted graph gives the high stress portion of the curves marked by green dashed rectangular. The black arrows show a series of serrated plastic flow events.

Fig. 3. (a) Fracture behaviors for as-cast specimen: (Left) Surface morphology and fracture angle; (Central) Magnified morphology for the portion marked by the red dashed circle in the left part of Fig. 3a; (Right) Fracture surface morphology. (b) Fracture behaviors for STS specimen I: (Left) Surface morphology and fracture angle; (Central) Magnified morphology for the STS portion marked by the red dashed circle in the left part of Fig. 3b; (Right) Fracture surface morphology. (c) Fracture behaviors for STS specimen II: (Left) Surface morphology and fracture angle; (Central) 
Magnified morphology for the STS portion marked by the red dashed circle in the left part of Fig. 3c; (Right) Fracture surface morphology. The black arrows points the multiple shear bands in the STS portion for STS specimen II.

Fig. 4. Finite element analysis of stress field distribution for STS specimen I and II under uniaxial tensile loading. Left: STS specimen II; Right: STS specimen I. (a) Whole stress field distribution. The grown arrows stand for the direction of extra tensile loading. 1, 2, 3, 4, 1', 2', 3' and 4' represent respectively the transverse sections of STS sample II and I. (b) Longitudinal sectional view of stress field of STS sample II and I in Fig. 4a. (c) Transverse sectional view of stress field corresponding to the transverse sections of 1, 2, 3, 4, 1', 2', 3' and 4' in Fig. 4a. The red arrows in the left part stand for the torsion direction of the twisted stress field for STS specimen II.

Table 1. Tensile fracture features for as-cast and STS specimen I and II: STS depth $d$, STS spacing $s$, STS width $w$, STS inclination angle $\theta_{s}$, fracture strength $\sigma_{f}$, plastic strain $\varepsilon_{p}$, fracture angle $\theta$, typical fracture morphology and fracture mode.

\begin{tabular}{|c|c|c|c|c|c|c|c|c|c|}
\hline Specimen & $d(\mu \mathrm{m})$ & $s(\mu \mathrm{m})$ & $w(\mu \mathrm{m})$ & $\theta_{s}\left(^{\circ}\right)$ & $\sigma_{f}(\mathrm{MPa})$ & $\varepsilon_{p}(\%)$ & $\theta\left(^{\circ}\right)$ & $\begin{array}{l}\text { Fracture } \\
\text { morphology }\end{array}$ & $\begin{array}{c}\text { Fracture } \\
\text { mode }\end{array}$ \\
\hline As-cast & 0 & 0 & 0 & 0 & 1614 & 0 & 53 & $\begin{array}{l}\text { Actinomorphic } \\
\text { pattern }\end{array}$ & Brittle \\
\hline STS I & 17 & 78 & 72 & 8.1 & 1590 & 0.002 & 55 & $\begin{array}{c}\text { Actinomorphic } \\
\text { pattern }\end{array}$ & Brittle \\
\hline STS II & 35 & 74 & 71 & 7.5 & 1506 & 0.90 & 66.1 & $\begin{array}{l}\text { River-like and } \\
\text { radial-like mixed } \\
\text { pattern }\end{array}$ & $\begin{array}{l}\text { Plastic, } \\
\text { serrated } \\
\text { flows }\end{array}$ \\
\hline
\end{tabular}




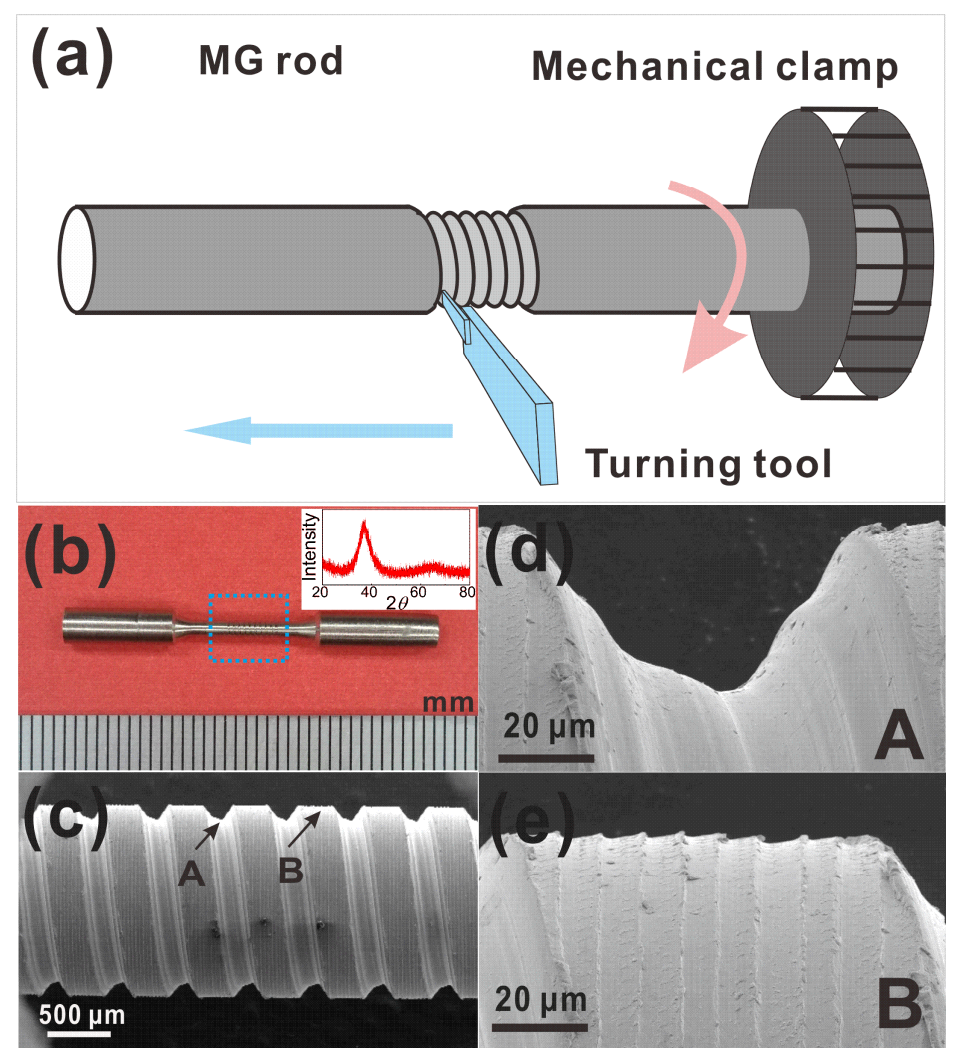

Fig. 1, Gao et al. 


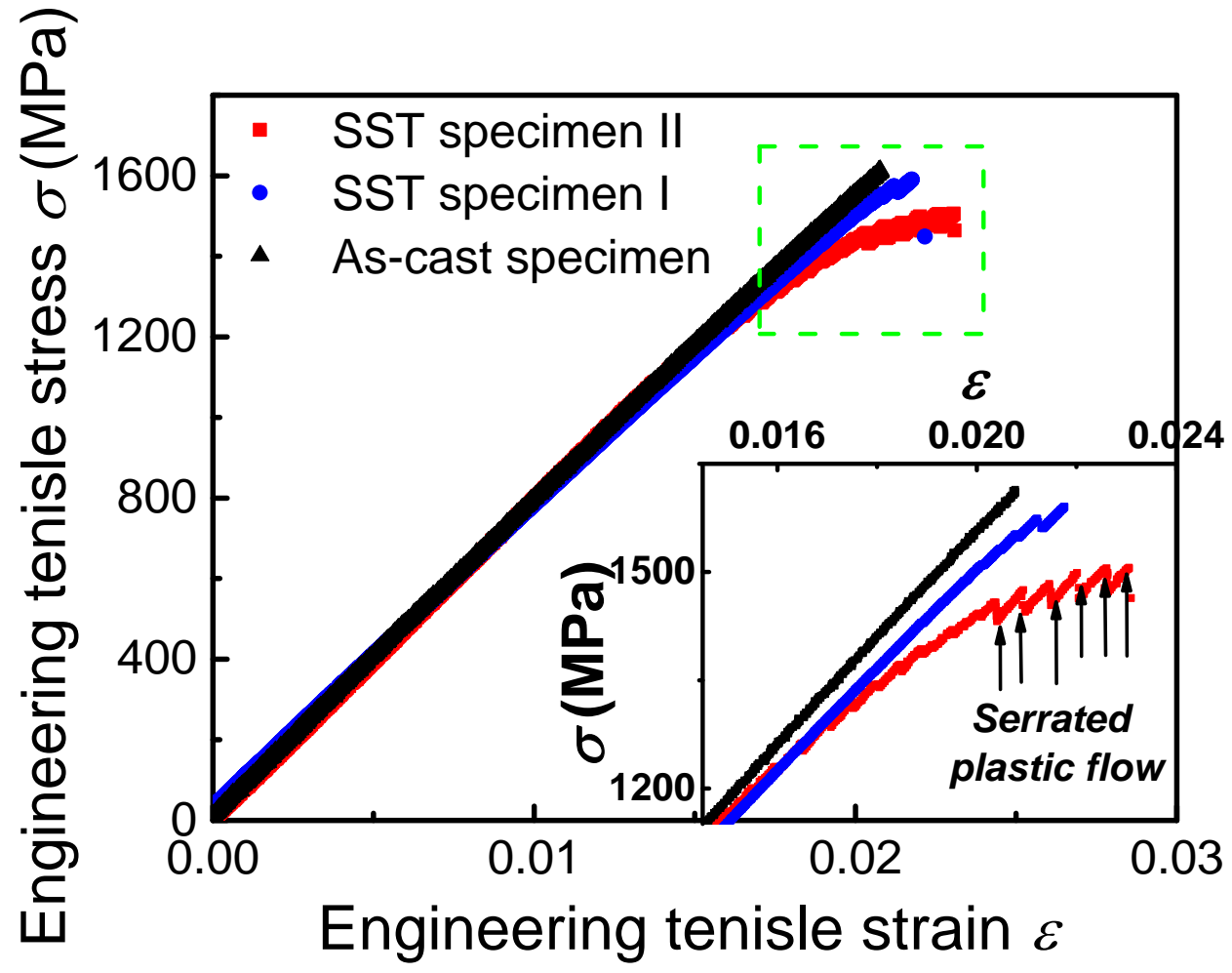

Fig. 2, Gao et al. 


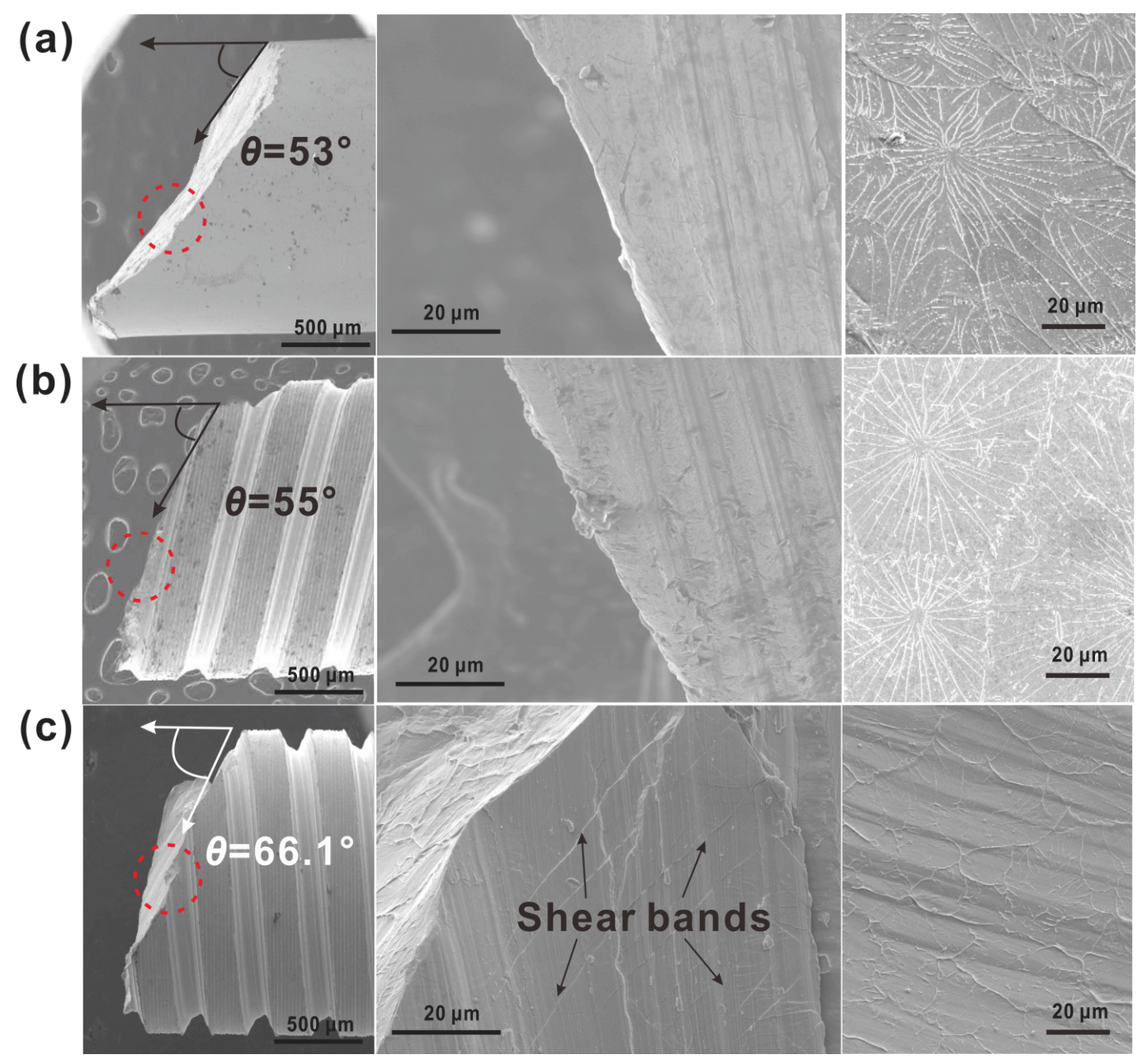

Fig. 3, Gao et al. 
(a)

STS specimen II

S, Mises, MPa

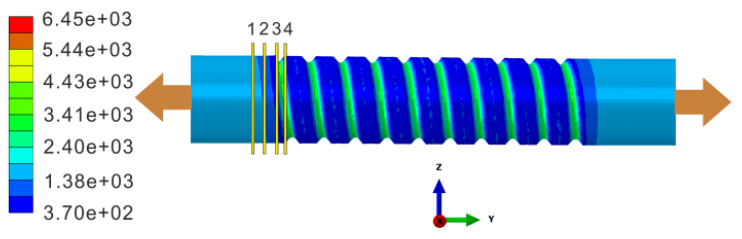

(b)

Longitudinal sectional view (Z-Y plane)

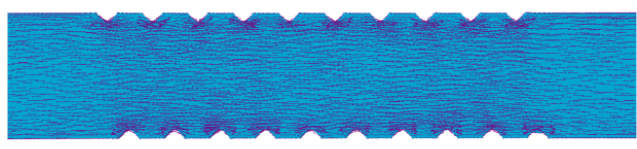

(c) Transverse sectional view (Z-X plane)

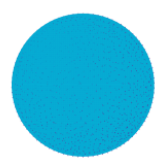

1

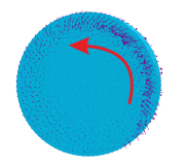

2

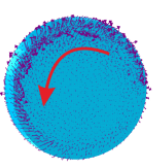

3

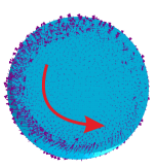

4
STS specimen I

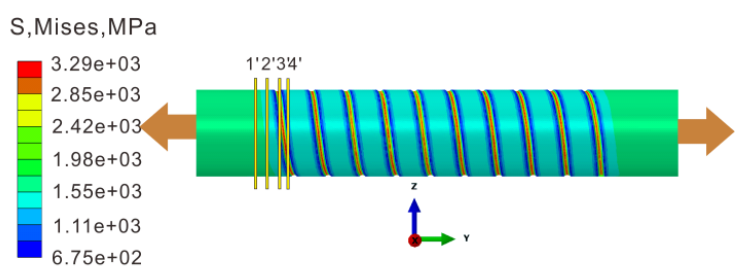

Longitudinal sectional view (Z-Y plane)

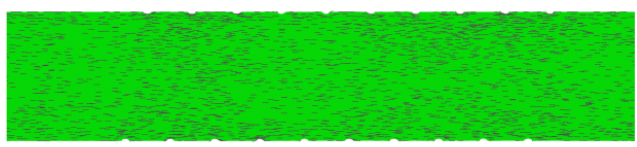

Transverse sectional view (Z-X plane)

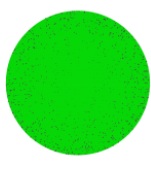

$1^{\prime}$

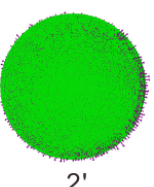

$2^{\prime}$

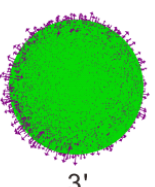

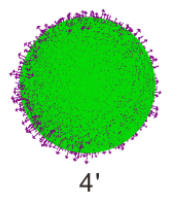

Fig. 4, Gao et al. 